\title{
On families between the Hardy-Littlewood and spherical maximal functions
}

\author{
Georgios Dosidis and Loukas Grafakos
}

\begin{abstract}
We study a family of maximal operators that provides a continuous link connecting the Hardy-Littlewood maximal function to the spherical maximal function. Our theorems are proved in the multilinear setting but may contain new results even in the linear case. For this family of operators we obtain bounds between Lebesgue spaces in the optimal range of exponents.
\end{abstract}

\section{Introduction}

Spherical averages arise naturally in PDE but $L^{p}$ bounds for maximal spherical averages were first obtained by Stein [24], who showed that the spherical maximal function

$$
S(f)(x):=\sup _{t>0} \frac{1}{\omega_{n-1}} \int_{\mathbb{S}^{n-1}}|f(x-t \theta)| d \sigma_{n-1}(\theta)
$$

is bounded from $L^{p}\left(\mathbb{R}^{n}\right)$ to $L^{p}\left(\mathbb{R}^{n}\right)$ when $p>\frac{n}{n-1}$ and $n \geq 3$ and is unbounded when $p \leq \frac{n}{n-1}$ and $n \geq 2$. The positive direction of this result was later extended to the case $n=2$ by Bourgain [4]. Here $d \sigma_{n-1}$ is the canonical surface measure on the sphere and $\omega_{n-1}$ is the measure of the entire unit sphere. A number of other authors have also studied the spherical maximal function; see for instance [6], [7], [20] and [23]. Extensions of the spherical maximal function to different settings have also been considered; see [5], [9], [14] and [19].

The boundedness of the maximal operator $S$ in [24] was obtain via the auxiliary family of operators

$$
S_{\alpha}(f)(x)=\sup _{t>0} \frac{2}{\omega_{n-1} B\left(\frac{n}{2}, 1-\alpha\right)} \int_{\mathbb{B}^{n}}|f(x-t y)|\left(1-|y|^{2}\right)^{-\alpha} d y,
$$

The authors acknowledge the support of the Simons Foundation (Grant 624733). The second author acknowledges the Simons Fellowship 819503. 
defined originally for Schwartz functions, where $0 \leq \alpha<1$. Here $\mathbb{B}^{n}$ is the unit ball in $\mathbb{R}^{n}, B$ is the beta function defined by $B(x, y)=\int_{0}^{1} t^{x-1}(1-t)^{y-1} d t$ for $x, y>0$. For each $0<\alpha<1$, Stein obtained boundedness for the operator $S_{\alpha}$ from $L^{p}$ to itself in the optimal range of exponents: $p>\frac{n}{n-\alpha}$, when $n \geq 3$. This was extended to the case $n=2$ indirectly in [4] and more explicitly in [20]. In [16] the authors obtained boundedness results for maximal operators associated to a more general set of measures that includes the family $S_{\alpha}$. function

Recall another classical averaging operator, the Hardy-Littlewood maximal

$$
M(f)(x)=\sup _{t>0} \frac{1}{v_{n}} \int_{\mathbb{B}^{n}}|f(x-t y)| d y .
$$

Here $f$ is a locally integrable function on $\mathbb{R}^{n}$ and $v_{n}$ is the volume of $\mathbb{B}^{n}$.

The relationship between the aforementioned operators is as follows: The family $S_{\alpha}$ provides a continuous link that connects $M$ to $S$ in the following explicit way: For any $f \in L_{l o c}^{1}\left(\mathbb{R}^{n}\right)$ and any $x \in \mathbb{R}^{n}$ we have

$$
\begin{aligned}
& M(f)(x) \leq S_{\alpha}(f)(x) \leq S(f)(x), \\
& \lim _{\alpha \rightarrow 1^{-}} S_{\alpha}(f)(x)=S(f)(x), \\
& \lim _{\alpha \rightarrow 0^{+}} S_{\alpha}(f)(x)=M(f)(x) .
\end{aligned}
$$

These assertions are contained in Theorem 2 and are proved in the next section.

In this paper, we denote by $d \sigma_{\varkappa-1}$ the surface measure on unit sphere $\mathbb{S}^{\varkappa-1}$ in $\mathbb{R}^{\varkappa}, v_{\varkappa}$ the measure of the unit ball in $\mathbb{R}^{\varkappa}$ and $\omega_{\varkappa-1}=d \sigma_{\varkappa-1}\left(\mathbb{S}^{\varkappa-1}\right)$ is the total measure of $\mathbb{S}^{\varkappa-1}$. Recall that $\varkappa v_{\varkappa}=\omega_{\varkappa-1}$ for any integer $\varkappa \geq 2$. We also use the notation $\mathbb{B}^{\varkappa}$ for the unit ball in $\mathbb{R}^{\varkappa}$ and $R \mathbb{B}^{\varkappa}$ for the ball of radius $R>0$ centered at the origin in $\mathbb{R}^{\varkappa}$. The space of Schwartz functions on $\mathbb{R}^{\varkappa}$ is denoted by $\mathcal{S}\left(\mathbb{R}^{\varkappa}\right)$.

Our purpose is to study multilinear versions of $S, S_{\alpha}$ and of $M$. We define a multi(sub)linear maximal operator as follows:

$$
M^{m}\left(f_{1}, \ldots, f_{m}\right)(x)=\sup _{t>0} \frac{1}{v_{m n}} \int_{\mathbb{B}^{m n}} \prod_{i=1}^{m}\left|f_{i}\left(x-t y_{i}\right)\right| d y_{1} \ldots d y_{m} .
$$

The uncentered version of this maximal operator first appeared in the work of Lerner, Ombrosi, Perez, Torres, Trujillo-Gonzalez [18] with the unit cube in place of the unit ball. Next, we introduce the family of operators

$$
S_{\alpha}^{m}\left(f_{1}, \ldots, f_{m}\right)(x)=\frac{2 / \omega_{m n-1}}{B\left(\frac{m n}{2}, 1-\alpha\right)} \sup _{t>0} \int_{\mathbb{B}^{m n}} \prod_{i=1}^{m}\left|f_{i}\left(x-t y_{i}\right)\right| \frac{d y}{\left(1-|y|^{2}\right)^{\alpha}}
$$

defined initially for functions $f_{i} \in \mathcal{S}\left(\mathbb{R}^{n}\right)$ and $0 \leq \alpha<1$. This is a multilinear extension of the operator $S_{\alpha}=S_{\alpha}^{1}$ introduced in (2). 
We recall the definition of the multilinear spherical maximal operator

$$
S^{m}\left(f_{1}, \ldots, f_{m}\right)(x)=\sup _{t>0} \frac{1}{\omega_{m n-1}} \int_{\mathbb{S}^{m n-1}} \prod_{i=1}^{m}\left|f_{i}\left(x-t \theta_{i}\right)\right| d \sigma_{m n-1}\left(\theta_{1}, \ldots, \theta_{m}\right),
$$

given also for functions $f_{i} \in \mathcal{S}\left(\mathbb{R}^{n}\right)$. When $m=1, S^{m}$ reduces to $S$ in (1). The bilinear analogue of Stein's spherical maximal function (when $\mathrm{m}=2$ ) was first introduced in [10] by Geba, Greenleaf, Iosevich, Palsson, and Sawyer who obtained the first bounds for it but later improved bounds were provided by [3], [12], [15] and [17]. A multilinear (non-maximal) version of this operator when all input functions lie in the same space $L^{p}(\mathbb{R})$ was previously studied by Oberlin [21]. The authors in [3] provided an example that shows that the bilinear spherical maximal function is not bounded when $p \geq \frac{n}{2 n-1}$. Last year Jeong and Lee in [17] proved that the bilinear maximal function is pointwise bounded by the product of the linear spherical maximal function and the Hardy-Littlewood maximal function, which helped them establish boundedness in the optimal open set of exponents, along with some endpoint estimates. These results were extended to the multilinear setting in [8]. Recently certain analogous bounds have been obtained by Anderson and Palsson in [1] and [2] concerning a discrete version of the multilinear spherical maximal function.

We would like to extend the definitions of the operators in (4) and (5) to functions in $f_{i}$ in $L_{l o c}^{1}\left(\mathbb{R}^{n}\right)$. Fix $f_{i}$ in $L_{l o c}^{1}\left(\mathbb{R}^{n}\right)$ and $x \in \mathbb{R}^{n}$; then

$$
t \longmapsto F(t)=t^{m n-1} \int_{\mathbb{S}^{m n-1}} \prod_{i=1}^{m}\left|f_{i}\left(x-t \theta_{i}\right)\right| d \sigma_{m n-1}\left(\theta_{1}, \ldots, \theta_{m}\right)
$$

is integrable over any interval $[0, L]$, which implies that the integrals in (5) are finite for almost all $t>0$. Likewise, if $F$ is as in $(6)$ and $t \in(0, L)$, then

$$
\int_{\mathbb{B}^{m n}} \prod_{i=1}^{m}\left|f_{i}\left(x-t y_{i}\right)\right| \frac{d y}{\left(1-|y|^{2}\right)^{\alpha}}=\int_{0}^{1} \frac{F(t r)}{\left(1-r^{2}\right)^{\alpha}} \frac{d r}{t^{m n-1}} \leq \frac{1}{t^{m n-\alpha}} \int_{0}^{L} \frac{F(s) d s}{(t-s)^{\alpha}}
$$

and the last integral is the convolution (evaluated at $t$ ) of the $L^{1}$ functions $F \chi_{[0, L]}$ and $s^{-\alpha} \chi_{(0, L]}$ on the real line, hence it is finite a.e. on $(0, L)$. We conclude that the integral in (4) is finite for almost all $t>0$ for $f_{i} \in L_{l o c}^{1}\left(\mathbb{R}^{n}\right)$ and $x \in \mathbb{R}^{n}$.

Now, one cannot properly define the supremum of a family $\left\{A_{t}\right\}_{t>0}\left(A_{t} \geq 0\right)$ which satisfies $A_{t}<\infty$ for almost all $t>0$. But it is possible to define the essential supremum of $\left\{A_{t}\right\}_{t>0}$, which is practically the supremum restricted over the subset of $(0, \infty)$ on which $A_{t}<\infty$. So to extend the definitions of the operators in $(5)$ and (4) to functions $f_{i} \in L_{l o c}^{1}\left(\mathbb{R}^{n}\right)$ for any $x \in \mathbb{R}^{n}$ by replacing the supremum in these expressions by the essential supremum ess.sup. However, this adjustment is not needed when $f_{i} \in L^{p_{i}}\left(\mathbb{R}^{n}\right)$ with $\sum_{i=1}^{n} \frac{1}{p_{i}}=\frac{1}{p}<\frac{m n-\alpha}{n}$, since, in that case, the 
corresponding averages vary continuously in $t$. See Corollary 5 below. Based on this discussion we provide the following definition.

Definition 1. Let $t>0, f_{i} \in L_{\text {loc }}^{1}\left(\mathbb{R}^{n}\right)$ for $1 \leq i \leq m$, and $x \in \mathbb{R}^{n}$. We define

$$
S_{\alpha, t}^{m}\left(f_{1}, \ldots, f_{m}\right)(x)=\frac{2 / \omega_{m n-1}}{B(m n / 2,1-\alpha)} \int_{\mathbb{B}^{m n}} \prod_{i=1}^{m} f_{i}\left(x-t y_{i}\right) \frac{d y}{\left(1-|y|^{2}\right)^{\alpha}}
$$

and

$$
S_{\alpha}^{m}\left(f_{1}, \ldots, f_{m}\right)(x)=\underset{t>0}{\operatorname{ess} . \sup } S_{\alpha, t}^{m}\left(\left|f_{1}\right|, \ldots,\left|f_{m}\right|\right)(x)
$$

for $0 \leq \alpha<1$. We also define

$$
S_{1, t}^{m}\left(f_{1}, \ldots, f_{m}\right)(x)=\frac{1}{\omega_{m n-1}} \int_{\mathbb{S}^{m n-1}} \prod_{i=1}^{m} f_{i}\left(x-t \theta_{i}\right) d \sigma_{m n-1}\left(\theta_{1}, \ldots, \theta_{m}\right)
$$

and

$$
S^{m}\left(f_{1}, \ldots, f_{m}\right)(x)=\underset{t>0}{\operatorname{ess.sup}} S_{1, t}^{m}\left(\left|f_{1}\right|, \ldots,\left|f_{m}\right|\right)(x) .
$$

In this paper we prove the following results:

Theorem 2. Let $0<\alpha<1$. Given $f_{i} \in L_{\text {loc }}^{1}\left(\mathbb{R}^{n}\right)$ and $x \in \mathbb{R}^{n}$ we have

$$
\begin{aligned}
& M^{m}\left(f_{1}, \ldots, f_{m}\right)(x) \leq S_{\alpha}^{m}\left(f_{1}, \ldots, f_{m}\right)(x) \leq S^{m}\left(f_{1}, \ldots, f_{m}\right)(x) \\
& \lim _{\alpha \rightarrow 1^{-}} S_{\alpha}^{m}\left(f_{1}, \ldots, f_{m}\right)(x)=S^{m}\left(f_{1}, \ldots, f_{m}\right)(x) \\
& \lim _{\alpha \rightarrow 0^{+}} S_{\alpha}^{m}\left(f_{1}, \ldots, f_{m}\right)(x)=M^{m}\left(f_{1}, \ldots, f_{m}\right)(x) .
\end{aligned}
$$

These statements are valid even when some of the preceding expressions equal $\infty$.

As $M^{m}$ is pointwise controlled by the product of the Hardy-Littlewood operators acting on each function, this operator is bounded from $L^{p_{1}}\left(\mathbb{R}^{n}\right) \times \ldots \times L^{p_{m}}\left(\mathbb{R}^{n}\right)$ to $L^{p}\left(\mathbb{R}^{n}\right)$ in the full range of exponents $1<p_{1}, \ldots, p_{m} \leq \infty$ and $1 / p_{1}+\ldots+1 / p_{m}=1 / p$. Boundedness for $S^{m}$ holds in the smaller region $n /(m n-1)<p \leq \infty$ as shown in [8]. So it is expected that $S_{\alpha}^{m}$ are bounded in some intermediate regions. This is the content of the following result.

Theorem 3. Let $n \geq 2,0 \leq \alpha<1$, and $1<p_{i} \leq \infty$. Define $p$ by $\sum_{i=1}^{m} \frac{1}{p_{i}}=\frac{1}{p}$. Then there is a constant $C=C\left(m, \alpha, p_{1}, \ldots, p_{m}\right)$ such that

$$
\left\|S_{\alpha}^{m}\left(f_{1}, \ldots, f_{m}\right)\right\|_{L^{p}\left(\mathbb{R}^{n}\right)} \leq C \prod_{i=1}^{m}\left\|f_{i}\right\|_{L^{p_{i}\left(\mathbb{R}^{n}\right)}}
$$

for all $f_{i} \in L^{p_{i}}\left(\mathbb{R}^{n}\right)$ if and only if

$$
\frac{n}{m n-\alpha}<p \leq \infty .
$$




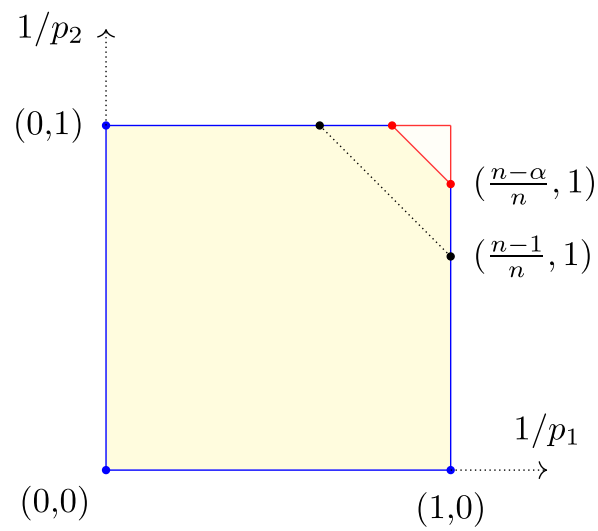

Figure 1. Range of $L^{p_{1}} \times L^{p_{2}} \rightarrow L^{p}$ boundedness of $S_{\alpha}^{2}$ when $0 \leq \alpha \leq 1$ and $n \geq 2$. The bilinear spherical maximal function is bounded below the black dotted line, while the bilinear HardyLittlewood maximal function is bounded on the entire square.

Moreover, if (15) holds, then the constant $C$ can be chosen to be independent of the dimension (as indicated by the parameters on which it is claimed to depend).

We graph the range of boundedness for the bilinear operator $S_{\alpha}^{2}$.

Remark 4. As a consequence, we obtain dimensionless $L^{p_{1}} \times \ldots \times L^{p_{m}} \rightarrow L^{p}$ bounds for the multilinear maximal function $M^{m}$ for all $\frac{1}{m}<p \leq \infty$; this extents the result of Stein and Strömberg [26] to the multilinear setting.

The estimates in (15) imply that when $f_{i} \in L^{p_{i}}\left(\mathbb{R}^{n}\right)$ with $\sum_{i=1}^{n} \frac{1}{p_{i}}=\frac{1}{p}<\frac{m n-\alpha}{n}$, then for almost all $x \in \mathbb{R}^{n}, S_{\alpha, t}^{m}\left(f_{1}, \ldots, f_{m}\right)(x)$ are finite uniformly in $t>0$.

Corollary 5. Let $0 \leq \alpha \leq 1$ and suppose that for all $1 \leq i \leq m, f_{i} \in L^{p_{i}}\left(\mathbb{R}^{n}\right)$ where $1<p_{i} \leq \infty$ satisfy $\sum_{i=1}^{m} \frac{1}{p_{i}}=\frac{1}{p}<\frac{m n-\alpha}{n}$. Then for almost every $x$ in $\mathbb{R}^{n}$, the function $t \mapsto S_{\alpha, t}^{m}\left(f_{1}, \ldots, f_{m}\right)(x)$ is well defined and continuous in $t \in(0, \infty)$. Therefore in Definition 1 , for almost all $x \in \mathbb{R}^{n}$, we can replace the essential supremum by a supremum in both (9) and (11).

Corollary 6. Let $0 \leq \alpha \leq 1$ and suppose that for all $1 \leq i \leq m, f_{i} \in L_{\text {loc }}^{p_{i}}\left(\mathbb{R}^{n}\right)$ where $1<p_{i} \leq \infty$ satisfy $\sum_{i=1}^{m} \frac{1}{p_{i}}=\frac{1}{p}<\frac{m n-\alpha}{n}$. Then for almost every $x \in \mathbb{R}^{n}$,

$$
\lim _{t \rightarrow 0} S_{\alpha, t}^{m}\left(f_{1}, \ldots, f_{m}\right)(x)=f_{1}(x) \ldots f_{m}(x) .
$$

Parts of Theorem 2 may be new even when $m=1$. Theorem 3 is only new when $m \geq 2$ as the case $m=1$ was considered in [24]. The proofs of these theorems can be suitably adapted to the measures

$$
\frac{q}{B(m n / q, 1-\alpha)} \frac{d \vec{y}}{\left(1-|\vec{y}|^{q}\right)^{\alpha}}
$$


for any $q>0$ in lieu of

$$
\frac{2}{B\left(\frac{m n}{2}, 1-\alpha\right)} \frac{d \vec{y}}{\left(1-|\vec{y}|^{2}\right)^{\alpha}}
$$

in (4). To simplify the notation in our proofs, we adopt the following conventions:

$$
\begin{array}{ll}
\vec{y}=\left(y_{1}, \ldots, y_{m}\right) \in\left(\mathbb{R}^{n}\right)^{m} & {[\vec{f}]=\left(f_{1}, \ldots, f_{m}\right)} \\
d \vec{y}=d y_{1} \ldots d y_{m} & \left(f_{1} \otimes \ldots \otimes f_{m}\right)(\vec{y})=f_{1}\left(y_{1}\right) \ldots f_{m}\left(y_{m}\right) \\
\vec{\theta}=\left(\theta_{1}, \ldots, \theta_{m}\right) \in \mathbb{S}^{m n-1} & \otimes \vec{f}=f_{1} \otimes \ldots \otimes f_{m} \\
\bar{x}=(\underbrace{(x, \ldots, x)}_{m \text { times }} \in\left(\mathbb{R}^{n}\right)^{m} & |\otimes \vec{f}|=\left|f_{1}\right| \otimes \ldots \otimes\left|f_{m}\right|
\end{array}
$$

Two main ideas are used in the proof of Theorem 2; integration by parts and the fundamental theorem of calculus, both with respect to the radial coordinate. Theorem 3 is based on a slicing formula that allows us to control $S_{\alpha}^{m}$ by the product of the Hardy-Littlewood maximal operators acting on $m-1$ input functions and of $S_{\alpha}$ (defined in (2)) acting on the remaining function. This gives estimates near the vertices of the region on which boundedness is claimed, while the remaining bounds are obtained by multilinear interpolation.

\section{The proof of Theorem 2}

Before we discuss the proof of Theorem 2 we note that when $\alpha=0$, equality holds in the first inequality in (12), since

$$
\frac{\omega_{m n-1}}{2} B\left(\frac{m n}{2}, 1\right)=\frac{\omega_{m n-1}}{2} \frac{2}{m n}=v_{m n} .
$$

That is, $M^{m}[\vec{f}](x)=S_{0}^{m}[\vec{f}](x)$. In fact (12) is valid even when $\alpha=0$, since

$$
\begin{aligned}
M^{m}[\vec{f}](x)=S_{0}^{m}[\vec{f}](x) & =\sup _{t>0} \frac{1}{v_{m n}} \int_{0}^{1} \int_{\mathbb{S}^{m n-1}}|\otimes \vec{f}|(\vec{x}-\operatorname{tr} \vec{\theta}) d \sigma_{m n-1}(\vec{\theta}) r^{m n-1} d r \\
& \leq \frac{1}{v_{m n}} \int_{0}^{1} \sup _{t^{\prime}>0} \int_{\mathbb{S}^{m n-1}}|\otimes \vec{f}|\left(\bar{x}-t^{\prime} \vec{\theta}\right) d \sigma_{m n-1}(\vec{\theta}) r^{m n-1} d r \\
& =m n S^{m}[\vec{f}](x) \int_{0}^{1} r^{m n-1} d r \\
& =S^{m}[\vec{f}](x) .
\end{aligned}
$$

Proof of Theorem 2. First we show that for any $0<\alpha<1$ we have $S_{\alpha}^{m}[\vec{f}](x) \leq$ $S^{m}[\vec{f}](x)$ for any $x \in \mathbb{R}^{n}$. Indeed, we have

$$
\frac{1}{\omega_{m n-1}} \frac{2}{B\left(\frac{m n}{2}, 1-\alpha\right)} \operatorname{ess.sup}_{t>0} \int_{\mathbb{B}^{m n}}|\otimes \vec{f}|(\bar{x}-t \vec{y})\left(1-|\vec{y}|^{2}\right)^{-\alpha} d \vec{y}
$$




$$
\begin{aligned}
& \leq \frac{1}{\omega_{m n-1}} \frac{2}{B\left(\frac{m n}{2}, 1-\alpha\right)} \int_{0}^{1} \frac{r^{m n-1}}{\left(1-r^{2}\right)^{\alpha}} \text { ess.sup } \int_{t>0}|\otimes \vec{f}|(\bar{x}-r t \vec{\theta}) d \sigma_{m n-1}(\vec{\theta}) d r \\
& \leq \frac{1}{\omega_{m n-1}} \frac{2}{B\left(\frac{m n}{2}, 1-\alpha\right)}\left(\int_{0}^{1} \frac{r^{m n-1}}{\left(1-r^{2}\right)^{\alpha}} d r\right) \underset{t^{\prime}>0}{\operatorname{ess.sup}} \int_{\mathbb{S}^{m n-1}}|\otimes \vec{f}|\left(\vec{x}-t^{\prime} \vec{\theta}\right) d \sigma(\vec{\theta}) \\
& =S^{m}[\vec{f}](x),
\end{aligned}
$$

as the $r$ integral in the parenthesis is equal to $\frac{1}{2} B\left(\frac{m n}{2}, 1-\alpha\right)$. This concludes the proof of the second inequality in (12).

Next we prove the first inequality in (12). That is, for a fixed $x \in \mathbb{R}^{n}$ and $0<\alpha<$ 1 , we show that $M^{m}[\vec{f}](x) \leq S_{\alpha}^{m}[\vec{f}](x)$. If for some $x \in \mathbb{R}^{n}$ we had $M^{m}[\vec{f}](x)=\infty$, we would also have that $S_{\alpha}^{m}[\vec{f}](x)=\infty$ as $\left(1-|\vec{y}|^{2}\right)^{-\alpha} \geq 1$ when $|\vec{y}|<1$. So we may assume that $M^{m}[\vec{f}](x)<\infty$ in the calculation below. For fixed $t>0$ we define

$$
H_{t}(r)=\int_{0}^{r} s^{m n-1}\left(\int_{\mathbb{S}^{m n-1}}|\otimes \vec{f}|(\bar{x}-t s \vec{\theta}) d \sigma(\vec{\theta})\right) d s=\int_{|\vec{y}| \leq r}|\otimes \vec{f}|(\bar{x}-t \vec{y}) d \vec{y},
$$

for $r>0$. As each $f_{j}$ is locally integrable, the integral on the right converges absolutely, and thus the expressions in the parentheses are finite for almost all $s>0$ and moreover, the $s$-integral converges absolutely. Thus $H_{t}(r)$ is the integral from 0 to $r$ of an $L^{1}$ function. Then, the Lebesgue differentiation theorem gives

$$
\frac{d}{d r} H_{t}(r)=H_{t}^{\prime}(r)=r^{m n-1} \int_{\mathbb{S}^{m n-1}}|\otimes \vec{f}|(\bar{x}-\operatorname{tr} \vec{\theta}) d \sigma(\vec{\theta}) \quad \text { for almost all } r>0 .
$$

Moreover, for any $r>0$ we have

$$
\underset{t>0}{\operatorname{ess.sup}} \frac{1}{v_{m n} r^{m n}} H_{t}(r)=\underset{t>0}{\operatorname{ess.sup}} \frac{1}{v_{m n}} H_{r t}(1)=\underset{t^{\prime}>0}{\operatorname{ess.sup}} \frac{1}{v_{m n}} H_{t^{\prime}}(1)=M^{m}[\vec{f}](x)<\infty
$$

where in the last equality we replaced the essential supremum by the supremum, using the continuity of the function

$$
t \longmapsto M_{t}^{m}\left(f_{1}, \ldots, f_{m}\right)(x)=\frac{1}{v_{m n}} \int_{\mathbb{B}^{m n}} \prod_{i=1}^{m}\left|f_{i}\left(x-t y_{i}\right)\right| d y_{1} \ldots d y_{m},
$$

for any $f_{i} \in L_{l o c}^{1}\left(\mathbb{R}^{n}\right)$, which can be obtained by an application of the Lebesgue dominated convergence theorem. Let

$$
c_{m n, \alpha}=\frac{2}{\omega_{m n-1} B\left(\frac{m n}{2}, 1-\alpha\right)} .
$$


For any $0<b<1$ we write

$$
\begin{aligned}
S_{\alpha}^{m} & {[\vec{f}](x) } \\
& \geq \underset{t>0}{\operatorname{ess.sup}} c_{m n, \alpha} \int_{0}^{b} H_{t}^{\prime}(r) \frac{1}{\left(1-r^{2}\right)^{\alpha}} d r \\
& =\underset{t>0}{\operatorname{ess.sup}} c_{m n, \alpha}\left[H_{t}(b) \frac{1}{\left(1-b^{2}\right)^{\alpha}}-\int_{0}^{b} H_{t}(r) \frac{-2 \alpha r}{\left(1-r^{2}\right)^{\alpha+1}} d r\right] \\
& \geq \underset{t>0}{\operatorname{ess.sup}} c_{m n, \alpha}\left[H_{t}(b) \frac{1}{\left(1-b^{2}\right)^{\alpha}}-\int_{0}^{b} M^{m}[\vec{f}](x) \frac{-2 \alpha r}{\left(1-r^{2}\right)^{\alpha+1}} v_{m n} r^{m n} d r\right] \\
& =c_{m n, \alpha}\left[M^{m}[\vec{f}](x) \frac{v_{m n} b^{m n}}{\left(1-b^{2}\right)^{\alpha}}-\int_{0}^{b} M^{m}[\vec{f}](x) \frac{-2 \alpha r}{\left(1-r^{2}\right)^{\alpha+1}} v_{m n} r^{m n} d r\right] \\
& =c_{m n, \alpha} M^{m}[\vec{f}](x) v_{m n}\left[\frac{b^{m n}}{\left(1-b^{2}\right)^{\alpha}}-\int_{0}^{b} \frac{-2 \alpha r}{\left(1-r^{2}\right)^{\alpha+1}} r^{m n} d r\right] \\
& =c_{m n, \alpha} M^{m}[\vec{f}](x) v_{m n}\left[m n \int_{0}^{b}\left(1-r^{2}\right)^{-\alpha} r^{m n-1} d r\right]
\end{aligned}
$$

where all the previous steps make use of the assumption that $M^{m}[\vec{f}](x)<\infty$. Letting $b \rightarrow 1^{-}$we obtain the first inequality in (12). So we established both inequalities in $(12)$ for $f_{i} \in L_{l o c}^{1}\left(\mathbb{R}^{n}\right)$.

Our next goal is to show that

$$
\lim _{\alpha \rightarrow 1^{-}} S_{\alpha}^{m}[\vec{f}](x) \geq S^{m}[\vec{f}](x),
$$

where $\underline{\lim }$ denotes the limit inferior. Let us fix $f_{j}$ in $L_{l o c}^{1}\left(\mathbb{R}^{n}\right)$ and $x \in \mathbb{R}^{n}$. We define

$$
G_{\vec{f}}(t)=\int_{\mathbb{S}^{m n-1}}|\otimes \vec{f}|(\bar{x}-t \vec{\theta}) d \sigma_{m n-1}(\vec{\theta}) .
$$

We observed earlier that for any $L<\infty$ we have

$$
\int_{0}^{L} t^{m n-1} G_{\vec{f}}(t) d t \leq \prod_{i=1}^{m} \int_{(|x|+1) \mathbb{B}^{n}}\left|f_{i}\left(y_{i}\right)\right| d y_{i}<\infty
$$

thus $G_{\vec{f}}(t)<\infty$ for almost all $t>0$. So let us fix a $t>0$ for which $G_{\vec{f}}(t)<\infty$. For this $t$ we will show that

$$
\lim _{\alpha \rightarrow 1^{-}} \int_{0}^{1} G_{\vec{f}}(r t) \frac{2 r^{m n-1}\left(1-r^{2}\right)^{-\alpha}}{B\left(\frac{m n}{2}, 1-\alpha\right)} d r=G_{\vec{f}}(t) .
$$


Once (18) is shown, we deduce

$$
\underline{\lim _{\alpha \rightarrow 1^{-}}} \sup _{t^{\prime}>0} \int_{0}^{1} G_{\vec{f}}\left(r t^{\prime}\right) \frac{2 r^{m n-1}\left(1-r^{2}\right)^{-\alpha}}{B\left(\frac{m n}{2}, 1-\alpha\right)} d r \geq G_{\vec{f}}(t)
$$

and taking the supremum on the right over all $t>0$ for which $G_{\vec{f}}(t)<\infty$, yields $(17)$. Notice that the supremum over these $t$ 's is the essential supremum which appears in the definition of this operator.

To prove (18), it will suffice to show that

$$
\lim _{\alpha \rightarrow 1^{-}} \int_{0}^{1}\left|G_{\vec{f}}(r t)-G_{\vec{f}}(t)\right| \frac{2 r^{m n-1}\left(1-r^{2}\right)^{-\alpha}}{B\left(\frac{m n}{2}, 1-\alpha\right)} d r=0 .
$$

For smooth functions with compact support $\varphi_{i}$ we have

$$
\lim _{\alpha \rightarrow 1^{-}} \int_{0}^{1}\left|G_{\vec{\varphi}}(r t)-G_{\vec{\varphi}}(t)\right| \frac{2 r^{m n-1}\left(1-r^{2}\right)^{-\alpha}}{B\left(\frac{m n}{2}, 1-\alpha\right)} d r=0
$$

as

$$
\left|\prod_{i=1}^{m}\right| \varphi_{i}\left(x-r t \theta_{i}\right)\left|-\prod_{i=1}^{m}\right| \varphi_{i}\left(x-t \theta_{i}\right)|| \leq\left|\prod_{i=1}^{m} \varphi_{i}\left(x-r t \theta_{i}\right)-\prod_{i=1}^{m} \varphi_{i}\left(x-t \theta_{i}\right)\right| \leq C t(1-r)
$$

and this factor cancels the singularity of $\left(1-r^{2}\right)^{-\alpha}$ while $\lim _{\alpha \rightarrow 1^{-}} B\left(\frac{m n}{2}, 1-\alpha\right)=$ $+\infty$.

Let us suppose that $0<\varepsilon<1$ is given. For our given $f_{i} \in L_{l o c}^{1}\left(\mathbb{R}^{n}\right)$, fixed $t>0$, and $x \in \mathbb{R}^{n}$, we pick $\varphi_{i}$ smooth functions with compact support such that

$$
\left\|f_{i}-\varphi_{i}\right\|_{L^{1}\left(\left(\frac{1}{t}+1\right)(|x|+1) \mathbb{B}^{n}\right)} \leq \varepsilon .
$$

This implies that

$$
\left\|f_{1} \otimes \ldots \otimes f_{m}-\varphi_{1} \otimes \ldots \otimes \varphi_{m}\right\|_{L^{1}\left(\left(\frac{1}{t}+1\right)(|x|+1) \mathbb{B}^{m n}\right)} \leq C^{\prime} \varepsilon,
$$

where

$$
C^{\prime}=\sum_{i=1}^{m} \prod_{\substack{1 \leq j \leq m \\ j \neq i}}\left(\left\|f_{j}\right\|_{L^{1}\left(\left(\frac{1}{t}+1\right)(|x|+1) \mathbb{B}^{n}\right)}+1\right)
$$

using the identity (valid for complex numbers $a_{i}, b_{i}$ )

$$
a_{1} a_{2} \ldots a_{m}-b_{1} b_{2} \ldots b_{m}=\sum_{i=1}^{m} b_{1} \ldots b_{i-1}\left(a_{i}-b_{i}\right) a_{i+1} \ldots a_{m} .
$$

In view of (20), the proof of (19) will be a consequence of the estimate:

$$
\int_{0}^{1}|Q(\vec{f}, \vec{\varphi}, t r, t)| \frac{2 r^{m n-1}\left(1-r^{2}\right)^{-\alpha}}{B\left(\frac{m n}{2}, 1-\alpha\right)} d r \leq C^{\prime \prime} \varepsilon
$$


where

$$
Q(\vec{f}, \vec{\varphi}, t r, t)=\left(G_{\vec{f}}(r t)-G_{\vec{f}}(t)\right)-\left(G_{\vec{\varphi}}(r t)-G_{\vec{\varphi}}(t)\right) .
$$

Notice that this function is integrable in $r$ over $[0,1]$. Thus the fundamental theorem of calculus applies, in the sense that $r^{K} Q(\vec{f}, \vec{\varphi}, t r, t)=\frac{d}{d r} \int_{0}^{r} s^{K} Q(\vec{f}, \vec{\varphi}, t s, t) d s$ for almost all $r$ in $[0,1]$. ( $K$ here is a fixed positive power.)

For any $0<b<1$ we have

$$
\begin{aligned}
& \int_{0}^{b}|Q(\vec{f}, \vec{\varphi}, t r, t)| \frac{2 r^{m n-1}\left(1-r^{2}\right)^{-\alpha}}{B\left(\frac{m n}{2}, 1-\alpha\right)} d r \\
= & \int_{0}^{b} \frac{d}{d r} \int_{0}^{r} s^{m n-1}|Q(\vec{f}, \vec{\varphi}, t s, t)| d s \frac{2\left(1-r^{2}\right)^{-\alpha}}{B\left(\frac{m n}{2}, 1-\alpha\right)} d r \\
= & \left(\int_{0}^{b} s^{m n-1}|Q(\vec{f}, \vec{\varphi}, t s, t)| d s\right) \frac{2\left(1-b^{2}\right)^{-\alpha}}{B\left(\frac{m n}{2}, 1-\alpha\right)} \\
& \quad-\int_{0}^{b}\left(\int_{0}^{r} s^{m n-1}|Q(\vec{f}, \vec{\varphi}, t s, t)| d s\right) \frac{2(-2 \alpha r)\left(1-r^{2}\right)^{-\alpha-1}}{B\left(\frac{m n}{2}, 1-\alpha\right)} d r \\
\leq & \left(\int_{0}^{1} s^{m n-1}|Q(\vec{f}, \vec{\varphi}, t s, t)| d s\right)\left[\frac{2\left(1-b^{2}\right)^{-\alpha}}{B\left(\frac{m n}{2}, 1-\alpha\right)}-\int_{0}^{b} \frac{2(-2 \alpha r)\left(1-r^{2}\right)^{-\alpha-1}}{B\left(\frac{m n}{2}, 1-\alpha\right)} d r\right] \\
= & \left(\int_{0}^{1} s^{m n-1}|Q(\vec{f}, \vec{\varphi}, t s, t)| d s\right)\left[\int_{0}^{b} \frac{2\left(1-r^{2}\right)^{-\alpha}}{B\left(\frac{m n}{2}, 1-\alpha\right)} d r\right] \\
\leq & \left(\int_{0}^{1} s^{m n-1}|Q(\vec{f}, \vec{\varphi}, t s, t)| d s\right)\left[\int_{0}^{b} \frac{2(1-r)^{-\alpha}}{B\left(\frac{m n}{2}, 1-\alpha\right)} d r\right] \\
\leq & \int_{0}^{1} s^{m n-1}|Q(\vec{f}, \vec{\varphi}, t s, t)| d s 2^{m n-1},
\end{aligned}
$$

as $(1-\alpha) B(m n, 1-\alpha)$ is bounded from below by some constant $C^{\prime}(m n)$. It remains to show that the integral

$$
\int_{0}^{1} s^{m n-1}|Q(\vec{f}, \vec{\varphi}, t s, t)| d s
$$

is bounded by a constant multiple of $\varepsilon$. But this integral is controlled by

$$
\begin{aligned}
\int_{\mathbb{B}^{m n}} & || \prod_{i=1}^{m} f_{i}\left(x-t y_{i}\right)|-| \prod_{i=1}^{m} \varphi_{i}\left(x-t y_{i}\right)|| d \vec{y} \\
& +\int_{\mathbb{B}^{m n}}|| \prod_{i=1}^{m} f_{i}\left(x-y_{i}\right)|-| \prod_{i=1}^{m} \varphi_{i}\left(x-y_{i}\right)|| d \vec{y}
\end{aligned}
$$


which, in turn, is bounded by

$$
\int_{\mathbb{B}^{m n}}\left|\prod_{i=1}^{m} f_{i}\left(x-t y_{i}\right)-\prod_{i=1}^{m} \varphi_{i}\left(x-t y_{i}\right)\right| d \vec{y}+\int_{\mathbb{B}^{m n}}\left|\prod_{i=1}^{m} f_{i}\left(x-y_{i}\right)-\prod_{i=1}^{m} \varphi_{i}\left(x-y_{i}\right)\right| d \vec{y} .
$$

Then using (23) we obtain that the preceding expression is bounded by $2 C^{\prime} \varepsilon$, where $C^{\prime}$ is as in (22). This proves (24), which as observed earlier, implies (17).

Finally, we prove (14). To do this, in view of (12), we fix $x$ in $\mathbb{R}^{n}$ and $f_{i}$ in $L_{l o c}^{1}\left(\mathbb{R}^{n}\right)$. It will suffice to show that

$$
\varlimsup_{\alpha \rightarrow 0^{+}} S_{\alpha}^{m}[\vec{f}](x) \leq M^{m}[\vec{f}](x) .
$$

For $t>0$ we set

$$
K_{t}(\alpha)=\frac{2}{\omega_{m n-1} B\left(\frac{m n}{2}, 1-\alpha\right)} \int_{\mathbb{B}^{m n}} \overrightarrow{|f|}(\bar{x}-t \vec{y})\left(1-|\vec{y}|^{2}\right)^{-\alpha} d \vec{y}
$$

Since we are taking the limit as $\alpha \rightarrow 0^{+}$we may consider $\alpha<1 / 2$. By the triangle inequality, for $0<\alpha<1 / 2$ we have

$$
K_{t}(\alpha) \leq K_{t}(0)+\left|K_{t}(\alpha)-K_{t}(0)\right| \leq K_{t}(0)+\alpha \sup _{0 \leq \beta \leq 1 / 2}\left|K_{t}^{\prime}(\beta)\right|
$$

where we denoted by $K_{t}^{\prime}(\beta)$ the derivative of $K_{t}$ with respect to $\beta$. Let us temporarily assume that $f_{i}$ are bounded functions. Fix $0 \leq \beta \leq 1 / 2$. We write

$$
\begin{aligned}
&\left|K_{t}^{\prime}(\beta)\right|= \frac{2 / \omega_{m n-1}}{B\left(\frac{m n}{2}, 1-\beta\right)^{2}}\left|B\left(\frac{m n}{2}, 1-\beta\right) \int_{\mathbb{B}^{m n}}\right| \otimes \vec{f} \mid(\bar{x}-t \vec{y})\left(\ln \frac{1}{1-|\vec{y}|^{2}}\right) \frac{d \vec{y}}{\left(1-|\vec{y}|^{2}\right)^{\beta}} \\
&-\left(\frac{d}{d \beta} B\left(\frac{m n}{2}, 1-\beta\right)\right) \int_{\mathbb{B}^{m n}}|\otimes \vec{f}|(\bar{x}-t \vec{y})\left(1-|\vec{y}|^{2}\right)^{-\beta} d \vec{y} \mid \\
& \leq \frac{2 \prod_{i=1}^{m}\left\|f_{i}\right\|_{L^{\infty}}}{B\left(\frac{m n}{2}, 1-\beta\right)} \int_{0}^{1}\left(1-r^{2}\right)^{-\beta} r^{m n-1}\left(\ln \frac{1}{1-r^{2}}\right) d r \\
& \quad+\frac{2 \prod_{i=1}^{m}\left\|f_{i}\right\|_{L^{\infty}}}{B\left(\frac{m n}{2}, 1-\beta\right)^{2}}\left(\int_{0}^{1} s^{-\beta}\left(\ln \frac{1}{s}\right)(1-s)^{\frac{m n}{2}-1} d s\right) \frac{1}{2} B\left(\frac{m n}{2}, 1-\beta\right) \\
& \leq \frac{2 \prod_{i=1}^{m}\left\|f_{i}\right\|_{L^{\infty}}}{B\left(\frac{m n}{2}, 1\right)}\left[\int_{0}^{1}\left(1-r^{2}\right)^{-\frac{1}{2}} r^{m n-1}\left(\ln \frac{1}{1-r^{2}}\right) d r\right. \\
&\left.\quad+\frac{1}{2} \int_{0}^{1} s^{-\frac{1}{2}}\left(\ln \frac{1}{s}\right)(1-s)^{\frac{m n}{2}-1} d s\right] \\
&=C_{\vec{f}, m n},
\end{aligned}
$$


where we used that $0 \leq \beta \leq 1 / 2$ and that

$$
\left|\frac{d}{d \beta} B\left(\frac{m n}{2}, 1-\beta\right)\right|=\int_{0}^{1} s^{-\beta}\left(\ln \frac{1}{s}\right)(1-s)^{\frac{m n}{2}-1} d s \leq \int_{0}^{1} s^{-\frac{1}{2}}\left(\ln \frac{1}{s}\right)(1-s)^{\frac{m n}{2}-1} d s .
$$

Taking the essential supremum in (26) with respect to $t>0$, we conclude for $\alpha<1 / 2$ that

$$
S_{\alpha}^{m}[\vec{f}](x) \leq M^{m}[\vec{f}](x)+\alpha C_{\vec{f}, m n} .
$$

Therefore for every $x \in \mathbb{R}^{n}$ we obtain

$$
\varlimsup_{\alpha \rightarrow 0^{+}} S_{\alpha}^{m}[\vec{f}](x) \leq M^{m}[\vec{f}](x),
$$

under the assumption that $f_{i}$ are bounded functions. We now remove this assumption on the $f_{i}$. Given $f_{i}$ in $L_{l o c}^{1}\left(\mathbb{R}^{n}\right)$, define $f_{i}^{k}=f_{i} \chi_{\left|f_{i}\right| \leq k}, k=1,2,3, \ldots$. Then

$$
\left|f_{i}^{1}\right| \leq\left|f_{i}^{2}\right| \leq\left|f_{i}^{3}\right| \leq \ldots \leq\left|f_{i}\right|
$$

$\left|f_{i}^{k}\right| \uparrow\left|f_{i}\right|$ as $k \rightarrow \infty$, and the functions $f_{i}^{k}$ are bounded. Let $\overrightarrow{\left[f^{k}\right]}=\left(f_{1}^{k}, \ldots, f_{m}^{k}\right)$. For each $k=1,2,3, \ldots$ and each $t>0$ the monotonicity of $S_{\alpha}^{m}$ in each variable and the preceding argument for bounded functions give

$$
\varlimsup_{\alpha \rightarrow 0^{+}} S_{\alpha}^{m}[\vec{f}](x) \geq \varlimsup_{\alpha \rightarrow 0^{+}} S_{\alpha}^{m} \overrightarrow{\left[f^{k}\right]}(x) \geq \frac{2 \omega_{m n-1}^{-1}}{B\left(\frac{m n}{2}, 1-\alpha\right)} \int_{\mathbb{B}^{m n}} \prod_{i=1}^{m}\left|f_{i}^{k}\left(x-t y_{i}\right)\right| \frac{d y}{\left(1-|y|^{2}\right)^{\alpha}} .
$$

Ignoring the middle term and letting $k \rightarrow \infty$ we obtain

$$
\varlimsup_{\alpha \rightarrow 0^{+}} S_{\alpha}^{m}[\vec{f}](x) \geq \frac{2 \omega_{m n-1}^{-1}}{B\left(\frac{m n}{2}, 1-\alpha\right)} \int_{\mathbb{B}^{m n}} \prod_{i=1}^{m}\left|f_{i}\left(x-t y_{i}\right)\right| \frac{d y}{\left(1-|y|^{2}\right)^{\alpha}}
$$

via the Lebesgue monotone convergence theorem. Taking the essential supremum over all $t>0$ yields inequality (25), and thus concludes the proof of (14).

\section{The proof of Theorem 3}

Proof of Theorem 3. For any $0 \leq \alpha<1$, we prove that the estimate

$$
S_{\alpha}^{m}\left(f_{1}, \ldots, f_{m}\right)(x) \leq S_{\alpha}\left(f_{k}\right)(x) \prod_{i \neq k} M\left(f_{i}\right)(x),
$$

is valid for all $f_{i} \in L_{l o c}^{1}\left(\mathbb{R}^{n}\right)$ and all $x \in \mathbb{R}^{n}$, where $S_{\alpha}$ is defined in (2) and $M$ is the Hardy-Littlewood maximal operator on $\mathbb{R}^{n}$. For any fixed $t>0$, we set

$$
S_{\alpha, t}^{m}\left(f_{1}, \ldots, f_{m}\right)(x)=c_{m n, \alpha} \int_{\mathbb{B}^{m n}} \prod_{i=1}^{m}\left|f_{i}\left(x-t y^{i}\right)\right|\left(1-|y|^{2}\right)^{-\alpha} d y
$$

where $c_{m n, \alpha}=2 /\left(\omega_{m n-1} B(m n / 2,1-\alpha)\right)$. 
For $y^{i} \in \mathbb{R}^{n}$ we set

$$
y=\left(y^{1}, \ldots, y^{m}\right) \quad \text { and } \quad \hat{y}^{k}=\left(y^{1}, \ldots, y^{k-1}, y^{k+1}, \ldots, y^{m}\right) .
$$

Then for a fixed $k \in\{1,2, \ldots, m\}$ we have

$$
\begin{aligned}
& c_{m n, \alpha}^{-1} S_{\alpha, t}^{m}\left(f_{1}, \ldots, f_{m}\right)(x) \\
& =\int_{\mathbb{B}^{m n}} \prod_{i=1}^{m}\left|f_{i}\left(x-t y^{i}\right)\right|\left(1-|y|^{2}\right)^{-\alpha} d y \\
& =\int_{\mathbb{B}^{(m-1) n}} \int_{\sqrt{1-\left|\hat{y}^{k}\right|^{2} \mathbb{B}^{n}}} \prod_{i=1}^{m}\left|f_{i}\left(x-t y^{i}\right)\right|\left(1-\left|\hat{y}^{k}\right|^{2}\right)^{-\alpha}\left(1-\left|\frac{y^{k}}{\sqrt{1-\left|\hat{y}^{k}\right|^{2}}}\right|^{2}\right)^{-\alpha} d y^{k} d \hat{y}^{k} \\
& =\int_{\mathbb{B}^{(m-1) n}} \prod_{i \neq k}\left|f_{i}\left(x-t y^{i}\right)\right| \int_{\mathbb{B}^{n}}\left|f_{k}\left(x-t \sqrt{1-\left|\hat{y}^{k}\right|^{2}} u^{k}\right)\right| \frac{\left(1-\left|\hat{y}^{k}\right|^{2}\right)^{\frac{n}{2}-\alpha}}{\left(1-\left|u^{k}\right|^{2}\right)^{\alpha}} d u^{k} d \hat{y}^{k} \\
& \leq \int_{\mathbb{B}^{(m-1) n}} \prod_{i \neq k}\left|f_{i}\left(x-t y^{i}\right)\right| \operatorname{ess.sup} \int_{\mathbb{B}^{n}}\left|f_{k}\left(x-t u^{k}\right)\right|\left(1-\left|u^{k}\right|^{2}\right)^{-\alpha} d u^{k} \frac{d \hat{y}^{k}}{\left(1-\left|\hat{y}^{k}\right|^{2}\right)^{\alpha-\frac{n}{2}}} \\
& \leq c_{n, \alpha}^{-1} S_{\alpha}\left(f_{k}\right)(x) \cdot \sup _{t>0} \int_{\mathbb{B}^{(m-1) n}} \prod_{i \neq k}\left|f_{i}\left(x-t y^{i}\right)\right| \frac{d \hat{y}^{k}}{\left(1-\left|\hat{y}^{k}\right|^{2}\right)^{\alpha-\frac{n}{2}}},
\end{aligned}
$$

with $c_{n, \alpha}=2 /\left(\omega_{n-1} B(n / 2,1-\alpha)\right)$.

Next, we use the following fact concerning multilinear approximate identities: Suppose that $\phi: \mathbb{R}^{\varkappa n} \rightarrow \mathbb{C}$ has an integrable radially decreasing majorant $\Phi$, and let $\phi_{t}(\vec{y})=t^{-\varkappa n} \phi(\vec{y} / t)$. If $*$ denotes convolution on $\mathbb{R}^{\varkappa n}$, then the estimate

$$
\sup _{t>0}\left|(\otimes \vec{f}) * \phi_{t}(\bar{x})\right| \leq\|\Phi\|_{L^{1}\left(\mathbb{R}^{\varkappa n}\right)} M^{m}[\vec{f}](x)
$$

is valid for all locally integrable functions $f_{j}$ on $\mathbb{R}^{n}, j=1, \ldots, \varkappa$. This follows by applying [11, Corollary 2.12] to the function $\left(x_{1}, \ldots, x_{\varkappa}\right) \mapsto \otimes \vec{f}\left(x_{1}, \ldots, x_{\varkappa}\right)$ on $\mathbb{R}^{\varkappa n}$ and using that the $\varkappa n$-dimensional Hardy-Littlewood maximal function of $\otimes \vec{f}$ at the point $(x, \ldots, x) \in\left(\mathbb{R}^{n}\right)^{\varkappa}$ equals $M^{m}[\vec{f}](x)$.

Returning to the previous calculation, for $\hat{y}^{k} \in \mathbb{R}^{(m-1) n}$ we consider the function $\phi\left(\hat{y}^{k}\right)=\left(1-\left|\hat{y}^{k}\right|^{2}\right)_{+}^{\frac{n}{2}-\alpha}$. Using that $n \geq 2$ (hence $n / 2-\alpha \geq 0$ ), we calculate that

$$
\|\phi\|_{L^{1}\left(\mathbb{R}^{(m-1) n}\right)}=\frac{\omega_{(m-1) n-1}}{2} B\left(\frac{(m-1) n}{2}, \frac{n}{2}+1-\alpha\right) .
$$

Using (28) for $\varkappa=m-1$, we can see that

$$
\sup _{t>0} \int_{\mathbb{B}^{(m-1) n}} \prod_{i \neq k}\left|f_{i}\left(x-t y^{i}\right)\right| \frac{d \hat{y}^{k}}{\left(1-\left|\hat{y}^{k}\right|^{2}\right)^{\alpha-\frac{n}{2}}} \leq\|\phi\|_{L^{1}\left(\mathbb{R}^{\varkappa n}\right)} M^{m-1}\left[\hat{f}^{k}\right](x),
$$


where $\left[\hat{f}^{k}\right]=\left(f_{1}, \ldots, f_{k-1}, f_{k+1}, f_{m}\right)$. Using the well known fact that $\omega_{n-1}=\frac{2 \pi^{n / 2}}{\Gamma(n / 2)}$ and the identity $B(a, b)=\frac{\Gamma(a) \Gamma(b)}{\Gamma(a+b)}$, one can verify that

$$
c_{m n, \alpha} \cdot c_{n, \alpha}^{-1} \cdot\|\phi\|_{L^{1}}=1 .
$$

Thus we conclude that

$$
S_{\alpha, t}^{m}\left(f_{1}, \ldots, f_{m}\right)(x) \leq S_{\alpha}\left(f_{k}\right)(x) M^{m-1}\left[\hat{f}^{k}\right](x) .
$$

Taking the essential supremum of $S_{\alpha, t}^{m}\left(f_{1}, \ldots, f_{m}\right)(x)$ over $t>0$ yields

$$
S_{\alpha}^{m}\left(f_{1}, \ldots, f_{m}\right)(x) \leq S_{\alpha}\left(f_{k}\right)(x) M^{m-1}\left[\hat{f}^{k}\right](x) .
$$

Since (29) holds for $\alpha=0$, we have that

$$
M_{\alpha}^{m}[\vec{f}] \leq M\left(f_{1}\right)(x) M^{m-1}\left[\hat{f}^{1}\right](x) .
$$

Therefore, consecutive applications of (29) conclude the proof of (27).

We now turn to the boundedness of $S_{\alpha}$ when $m=1$. It was shown in [24] that $S_{\alpha}$ is bounded on $L^{p}$ for $\frac{n}{n-\alpha}<p \leq \infty$ when $n \geq 3$. We remark that this result also holds when $n=2$. We now provide a sketch of a proof valid in all dimensions $n \geq 2$. To do this, for $f \in \mathcal{S}\left(\mathbb{R}^{n}\right)$, we express $S_{\alpha} f$ as a maximal multiplier operator

$$
S_{\alpha} f(x)=\frac{2 \pi^{\alpha}}{\omega_{n-1}} \frac{\Gamma(1-\alpha)}{B\left(\frac{n}{2}, 1-\alpha\right)} \sup _{t>0}\left|\int_{\mathbb{R}^{n}} \hat{f}(\xi) \frac{J_{\frac{n}{2}-\alpha}(2 \pi t|\xi|)}{|t \xi|^{\frac{n}{2}-\alpha}} e^{2 \pi i x \cdot \xi} d \xi\right|
$$

using the identity in [11, Appendix B.5]. To derive this we use the Bochner-Riesz multiplier $\left(1-|x|^{2}\right)^{-\alpha}$ with a negative exponent, viewed as a kernel. Then the Fourier transform expression for $\left(1-|x|^{2}\right)^{z}$ when $\operatorname{Re} z>0$ is also valid for $\operatorname{Re} z>-1$ by analytic continuation. Notice that in this range of $z$, the kernel remains locally integrable. Using properties of Bessel functions, the multiplier

$$
m_{\alpha}(\xi)=\frac{J_{\frac{n}{2}-\alpha}(2 \pi|\xi|)}{|\xi|^{\frac{n}{2}-\alpha}}
$$

is a smooth function which satisfies for all multi-indices $\gamma$

$$
\left|\partial_{\xi}^{\gamma} m_{\alpha}(\xi)\right| \leq \frac{C_{n, \gamma}}{|\xi|^{\frac{n+1}{2}-\alpha}}
$$

and the exponent $a=\frac{n+1}{2}-\alpha$ is strictly bigger than $\frac{1}{2}$ (since $n \geq 2$ and $\alpha<1$ ). Then the hypotheses of $\left[22\right.$, Theorem B] apply and we obtain that $S_{\alpha}$ is bounded on $L^{p}\left(\mathbb{R}^{n}\right)$ (when restricted to Schwartz functions) for

$$
p>\frac{2 n}{n+2 a-1}=\frac{n}{n-\alpha} \text {. }
$$


(In [22, Theorem B] there is an upper restriction on $p$, but as $S_{\alpha}$ is bounded on $L^{\infty}$ this does not apply here.) Then $S_{\alpha}$ extends to general $f \in L^{p}\left(\mathbb{R}^{n}\right)$ for $p>\frac{n}{n-\alpha}$ by density, and this extension coincides with that given in Definition 1.

We now use (27) to obtain that

$$
\left\|S_{\alpha}^{m}\left(f_{1}, \ldots, f_{m}\right)\right\|_{L^{p}\left(\mathbb{R}^{n}\right)} \leq C \prod_{i=1}^{m}\left\|f_{i}\right\|_{L^{p_{i}\left(\mathbb{R}^{n}\right)}}
$$

for all $f_{i} \in L^{p_{i}}$, when $1<p_{i} \leq \infty$ for $i \neq k$ and $\frac{n}{n-\alpha}<p_{k} \leq \infty$. Here the constant $C=$ $C\left(m, \alpha, p_{1}, \ldots, p_{m}\right)$ doesn't depend on the dimension $n$, since $S_{\alpha}(f)(x) \leq S_{1}(f)(x)$ and $\left\|S_{1}(f)(x)\right\|_{L^{p}\left(\mathbb{R}^{n}\right)} \leq c\|f\|_{L^{p}\left(\mathbb{R}^{n}\right)}$ for a constant $c$ independent of $n$ (see [26]).

To describe geometrically the points $\left(1 / p_{1}, \ldots, 1 / p_{m}\right)$ for which we claim boundedness for $S_{\alpha}^{m}$, consider the cube $Q=[0,1]^{m}$ and let $V$ be the set of all of its vertices except for the vertex $(1,1, \ldots, 1)$. Then $|V|=2^{m}-1$. We consider the intersection of $Q$ with the half-space $H$ of $\mathbb{R}^{m}$ described by

$$
H=\left\{\left(t_{1}, \ldots, t_{m}\right): t_{1}+\ldots+t_{m} \leq \frac{m n-\alpha}{n}\right\} .
$$

Then $Q \cap H$ has $2^{m}-1+m$ vertices, namely the set $V$ union the $m$ points

$$
\left(1, \ldots, 1, \frac{m n-\alpha}{n}, 1, \ldots, 1\right)
$$

where $\frac{m n-\alpha}{n}$ ranges over the $m$ slots. We claim that $S_{\alpha}^{m}$ satisfies strong $L^{p}$ bounds in the interior of $Q \cap H$. To see this, we interpolate between estimates at the vertices of $Q \cap H$. Precisely, the interpolation works as follows: Let $W$ be the vertices of $Q \cap H$ that do not belong to $V$ and let $W^{\prime}$ be a finite union of open balls centered at the points of $W$ intersected with $Q \cap H$. We interpolate between points $P=$ $\left(1 / p_{1}, \ldots, 1 / p_{m}\right)$ in $V \cup W^{\prime}$. If $P \in V$, then we have an estimate $L^{p_{1}} \times \ldots \times L^{p_{m}}$ to $L^{p}$ for $S_{\alpha}^{m}$, as at least one coordinate $1 / p_{k}$ is 0 (i.e., $p_{k}=\infty$ ), and we apply (30) for this $k$. Now if $P$ lies in $W^{\prime}$, then there is a $k \in\{1, \ldots, m\}$ such that $p_{k}>\frac{n}{n-\alpha}$ and $p_{i}$ are near 1 for all $i \neq k$. Using estimate (30) again for this choice of $k$, we obtain that $S_{\alpha}^{m}$ maps $L^{p_{1}} \times \ldots \times L^{p_{m}}$ to $L^{p}$ at this point $P$. Applying the $m$-linear version of the Marcinkiewicz interpolation theorem [13], we deduce the boundedness of $S_{\alpha}^{m}$ in the interior of $Q \cap H$. Similar reasoning provides weak type bounds on all the faces of $Q \cap H$, except possibly on the $H$ face, on which we don't know if there are any bounds at all.

Finally we show the optimality of the range $p>\frac{n}{m n-\alpha}$. We consider the action of $S_{\alpha}^{m}$ on characteristic functions; specifically, let $f_{1}=\ldots=f_{m}=\chi_{\mathbb{B}^{n}}$. Since the characteristic functions belong in all $L^{p}$ spaces, in the definition of $S_{\alpha}^{m}[\vec{f}]$ we can 
replace the essential supremum by the supremum (see Corollary 5). Therefore for $|x|$ sufficiently large it is enough to pick $t=\sqrt{m}|x|$ in order to write the estimate

$$
\begin{aligned}
c_{m n, \alpha}^{-1} S_{\alpha}^{m}\left(f_{1}, \ldots, f_{m}\right)(x) & \geq \int_{\mathbb{B}^{m n}} \prod_{i=1}^{m}\left|f_{i}\left(x-\sqrt{m}|x| y_{i}\right)\right| \cdot\left(1-|\vec{y}|^{2}\right)^{-\alpha} d \vec{y} \\
& \geq \int_{\left|\vec{y}-\frac{\bar{x}}{\sqrt{m}|x|}\right| \leq \frac{1}{\sqrt{m}|x|}}\left(1-|\vec{y}|^{2}\right)^{-\alpha} d \vec{y} \\
& \geq 2^{-\alpha} \int_{\left|\vec{y}-\frac{\bar{x}}{\sqrt{m}|x|}\right| \leq \frac{1}{\sqrt{m}|x|}}(1-|\vec{y}|)^{-\alpha} d \vec{y}
\end{aligned}
$$

since

$$
\left|\vec{y}-\frac{\bar{x}}{\sqrt{m}|x|}\right| \leq \frac{1}{\sqrt{m}|x|} \Longrightarrow|x-\sqrt{m}| x\left|y_{j}\right| \leq 1 \quad \text { for all } j=1, \ldots, m
$$

The point $\overline{\theta_{x}}=\frac{\bar{x}}{\sqrt{m}|x|}$ lies in the sphere $\mathbb{S}^{m n-1}$. A simple geometric argument gives that the integral in (31) expressed in polar coordinates $\vec{y}=r \vec{\theta}$ is at least

$$
\int_{1-\frac{c}{|x|}}^{1}(1-r)^{-\alpha} r^{m n-1} \int_{\left|\vec{\theta}-\overline{\theta_{x}}\right| \leq \frac{c}{|x|}} d \sigma_{m n-1}(\vec{\theta}) \frac{d r}{2^{\alpha}} \geq \frac{2^{-\alpha}}{|x|^{1-\alpha}} \frac{C(m, n)}{|x|^{m n-1}}=\frac{2^{-\alpha} C(m, n)}{|x|^{m n-\alpha}}
$$

for some small constants $c, C$ (depending on $n$ and $m$ ). We conclude the proof by noting that the function $|x|^{-m n+\alpha} \chi_{|x| \geq 100}$ does not lie in $L^{p}\left(\mathbb{R}^{n}\right)$ for $p \leq \frac{n}{m n-\alpha}$.

We proved Theorem 3 working directly with $L^{p_{i}}$ functions. Alternatively, we could have worked with a dense family of $L^{p_{i}}$ and then extend to $L^{p_{i}}$ by density. There is no ambiguity in this extension, in view of the following proposition.

Proposition 7. Let $0<p_{1}, \ldots, p_{m}, p \leq \infty$. Suppose that $T$ is a subadditive operator in each variable $\left(^{1}\right)$ that satisfies the estimate

$$
\left\|T\left(f_{1}, \ldots, f_{m}\right)\right\|_{L^{p}} \leq K\left\|f_{1}\right\|_{L^{p_{1}}} \ldots\left\|f_{m}\right\|_{L^{p_{m}}}
$$

for all functions $f_{j}$ in a dense subspace of $L^{p_{j}}$. Then $T$ admits a unique bounded subadditive extension from $L^{p_{1}} \times \ldots \times L^{p_{m}}$ to $L^{p}$ with the same bound.

Proof. For any $j \in\{1, \ldots, m\}$, given $f_{j} \in L^{p_{j}}$ pick sequences $a_{j}^{k}, b_{j}^{l}, k, l=1,2,3, \ldots$ in the given dense subspace of $L^{p_{j}}$ which converge to $f_{j}$ in $L^{p_{j}}$. Using the idea

$\left({ }^{1}\right)$ this means $|T(\ldots, f+g, \ldots)| \leq|T(\ldots, f, \ldots)|+T(\ldots, g, \ldots) \mid$ for all $f, g$ 
proving (23) and the subadditivity of $T$ in each variable we obtain:

$$
\begin{gathered}
\left|T\left(a_{1}^{k}, a_{2}^{k}, \ldots, a_{m}^{k}\right)-T\left(b_{1}^{l}, b_{2}^{l}, \ldots, b_{m}^{l}\right)\right| \leq \\
\sum_{i=1}^{m}\left[\left|T\left(b_{1}^{l}, \ldots, b_{i-1}^{l}, a_{i}^{k}-b_{i}^{l}, a_{i+1}^{k}, \ldots, a_{m}^{k}\right)\right|+\left|T\left(a_{1}^{k}, \ldots, a_{i-1}^{k}, b_{i}^{k}-a_{i}^{l}, b_{i+1}^{l}, \ldots, b_{m}^{l}\right)\right|\right] .
\end{gathered}
$$

Applying the $L^{p}$ (quasi norm) and hypothesis (32) we deduce

$$
\begin{aligned}
& \left\|T\left(a_{1}^{k}, a_{2}^{k}, \ldots, a_{m}^{k}\right)-T\left(b_{1}^{l}, b_{2}^{l}, \ldots, b_{m}^{l}\right)\right\|_{L^{p}} \\
& \quad \leq C_{p} K \sum_{i=1}^{m}\left\|a_{i}^{k}-b_{i}^{l}\right\|_{L^{p_{i}}} \prod_{j \neq i}\left[\left\|a_{j}^{k}\right\|_{L^{p_{j}}}+\left\|b_{j}^{l}\right\|_{L^{p_{j}}}\right] .
\end{aligned}
$$

Taking $b_{j}^{l}=a_{j}^{l}$ in (33) we conclude that the sequence $\left\{T\left(a_{1}^{k}, a_{2}^{k}, \ldots, a_{m}^{k}\right)\right\}_{k=1}^{\infty}$ is Cauchy in $L^{p}$ and thus it has a limit $\bar{T}\left(f_{1}, \ldots, f_{m}\right)$. This limit does not depend on the choice of the sequences $a_{j}^{k}$ converging to $f_{j}$, as we can choose $l=k$ in (33) and let $k \rightarrow \infty$. Thus $T$ has a unique extension $\bar{T}$. This extension is also bounded with the same bound and is subadditive by density.

\section{The proofs of Corollaries 5 and 6}

Next we discuss the proof of Corollary 5. The case $m=1$ of this result is contained in [25, Chapter XI Section 3.5].

Proof. It suffices to prove the assertion for almost all $x$ in a ball $N \mathbb{B}^{n}$, as $\mathbb{R}^{n}$ is a countable union of $N \mathbb{B}^{n}$ over $N=1,2, \ldots$. Let us fix such a ball $N \mathbb{B}^{n}$. It will suffice to prove the continuity of $t \mapsto S_{\alpha, t}\left(f_{1}, \ldots, f_{m}\right)(x)$ on $(0, R)$ for every $R>0$. Fix such an $R>0$ as well. Then we may replace each $f_{i}$ by $g_{i}=f_{i} \chi(N+R) \mathbb{B}^{n}$ as $S_{\alpha, t}\left(f_{1}, \ldots, f_{m}\right)(x)=S_{\alpha, t}\left(g_{1}, \ldots, g_{m}\right)(x)$ when $x \in N \mathbb{B}^{n}$ and $0<t<R$. As $g_{i}$ have compact support and lie in $L^{p_{i}}$, they also lie in $L^{q_{i}}$, where $q_{i}<p_{i}$ are chosen so that $\frac{1}{q}=\sum_{i=1}^{m} \frac{1}{q_{i}}<\frac{m n-\alpha}{n}$. (The purpose of introducing $q_{i}<p_{i}$ was to replace all infinite indices $p_{i}$ by finite ones, as there is no good dense subspace of $L^{\infty}$.)

We pick sequences $\varphi_{j}^{k}$ of smooth compactly supported functions with $\varphi_{j}^{k} \rightarrow g_{j}$ in $L^{q_{j}}\left(\mathbb{R}^{n}\right)$ (since $\left.q_{j}<\infty\right)$ and consider the sequence

$$
\underset{t>0}{\operatorname{ess.sup}} S_{\alpha, t}^{m}\left(g_{1}-\varphi_{1}^{k}, \ldots, g_{m}-\varphi_{m}^{k}\right), \quad m=1,2,3, \ldots
$$

By (15) if $\alpha<1$ (or by [8] if $\alpha=1$ ) this sequence converges to zero in $L^{q}\left(\mathbb{R}^{n}\right)$, thus there is a subsequence that converges to zero a.e. This implies that there is a subset $E$ of $\mathbb{R}^{n}$ of measure zero such that for all $x \in \mathbb{R}^{n} \backslash E$ we have

$$
\lim _{k \rightarrow \infty}\left\|S_{\alpha, t}^{m}[\vec{g}](x)-S_{\alpha, t}^{m} \overrightarrow{\left[\varphi^{k}\right]}(x)\right\|_{L^{\infty}((0, \infty), d t)}=0
$$


i.e., $S_{\alpha, t}^{m} \overrightarrow{\left[\varphi^{k}\right]}(x) \rightarrow S_{\alpha, t}^{m}[\vec{f}](x)$ uniformly in $t>0$. Since $S_{\alpha, t}^{m} \overrightarrow{\left[\varphi^{k}\right]}(x)$ is continuous in $t$, we conclude that $S_{\alpha, t}^{m}[\vec{g}](x)$ is also continuous in $t$, for almost every $x \in \mathbb{R}^{n}$.

To prove Corollary 6 , we will need a proposition analogous to [11, Theorem 2.1.14]. Let $(X, \mu)$ and $(Y, \nu)$ be $\sigma$ finite measure spaces and let $0<p_{j} \leq \infty, j=$ $1, \ldots, m$, and $0<q<\infty$. Let $D_{j}$ be a dense subspace of $L^{p_{j}}(X, \mu)$. Suppose that for all $t>0, T_{t}$ is an $m$-linear operator defined on $L^{p_{1}}(X, \mu) \times \ldots \times L^{p_{m}}(X, \mu)$ with values in the space of measurable functions defined a.e. on $Y$. Assume that for all $f_{j} \in L^{p_{j}}$, the function

is $\nu$-measurable on $Y$.

$$
y \longmapsto T_{*}\left(f_{1}, \ldots, f_{m}\right)(y)=\sup _{t>0}\left|T_{t}\left(f_{1}, \ldots, f_{m}\right)(y)\right|
$$

Proposition 8. Let $0<p_{i} \leq \infty, 1 \leq i \leq m, 0<q<\infty$ and $T_{t}$ and $T_{*}$ as in the previous discussion. Suppose that there is a constant $B$ such that

$$
\left\|T_{*}\left(f_{1}, \ldots, f_{m}\right)\right\|_{L^{q, \infty}} \leq B \prod_{j=1}^{m}\left\|f_{j}\right\|_{L^{p_{j}}}
$$

for all $f_{j} \in L^{p_{j}}(X, \mu)$. Also suppose that for all $\varphi_{j} \in D_{j}$

$$
\lim _{t \rightarrow 0} T_{t}\left(\varphi_{1}, \ldots, \varphi_{m}\right)=T\left(\varphi_{1}, \ldots, \varphi_{m}\right)
$$

exists and is finite $\nu$-a.e. Then for all functions $f_{j} \in L^{p_{j}}(X, \mu)$ the limit in (35) exists and is finite $\nu$-a.e., and defines an $m$-linear operator which uniquely extends $T$ defined on $D_{1} \times \ldots \times D_{m}$ and which is bounded from $L^{p_{1}} \times \ldots \times L^{p_{m}}$ to $L^{q, \infty}(X)$.

Proof. Given $f_{j} \in L^{p_{j}}$ we define the oscillation of $\vec{f}$ for $y \in Y$ by setting

$$
O_{\vec{f}}(y)=\limsup _{\varepsilon \rightarrow 0} \limsup _{\theta \rightarrow 0}\left|T_{\varepsilon}[\vec{f}](y)-T_{\theta}[\vec{f}](y)\right| \text {. }
$$

We will show that for all $f_{j} \in L^{p_{j}}$ and all $\delta>0$,

$$
\nu\left(\left\{y \in Y: O_{\vec{f}}(y)>\delta\right\}\right)=0 .
$$

Once (36) is established, we obtain that $O_{\vec{f}}(y)=0$ for $\nu$-almost all $y$, which implies that $T_{t}[\vec{f}](y)$ is Cauchy for $\nu$-almost all $y$, and it therefore converges $\nu$-a.e. to some $T[\vec{f}](y)$ as $t \rightarrow 0$. The operator $T$ defined this way on $L^{p_{1}}(X) \times \ldots \times L^{p_{m}}(X)$ is linear and extends $T$ given in (35) defined on $D_{1} \times \ldots \times D_{m}$.

To approximate $O_{\vec{f}}(y)$ we use density. Given $0<\eta<1$, we find $\varphi_{j} \in D_{j}$ such that $\left\|f_{j}-\varphi_{j}\right\|_{L^{p_{j}}}<\eta, j=1, \ldots, m$. Without a loss of generality, we also assume that $\left\|\varphi_{i}\right\|_{L^{p_{i}}} \leq 2\left\|f_{i}\right\|_{L^{p_{i}}}$. Since $T_{t}[\vec{\varphi}] \rightarrow T[\vec{\varphi}] \nu$-a.e., it follows that $O_{\vec{\varphi}}=0 \nu$-a.e. Using (23), we write

$$
T_{t}[\vec{f}]-T_{t}[\vec{\varphi}]=\sum_{i=1}^{m} T_{t}\left(\varphi_{1}, \ldots, \varphi_{i-1}, f_{i}-\varphi_{i}, f_{i+1}, \ldots, f_{m}\right)
$$


and from this we obtain

$$
O_{\vec{f}} \leq O_{\vec{\varphi}}+\sum_{i=1}^{m} O_{\left(\varphi_{1}, \ldots, \varphi_{i-1}, f_{i}-\varphi_{i}, f_{i+1}, \ldots, f_{m}\right)} \quad \nu \text {-a.e. }
$$

Now, for any $\delta>0$ we have

$$
\begin{aligned}
\nu & \left(\left\{y \in Y: O_{\vec{f}}(y)>\delta\right\}\right) \\
& \leq \nu\left(\left\{y \in Y: \sum_{i=1}^{m} O_{\left(\varphi_{1}, \ldots, \varphi_{i-1}, f_{i}-\varphi_{i}, f_{i+1}, \ldots, f_{m}\right)}>\delta\right\}\right) \\
& \leq \nu\left(\left\{y \in Y: \sum_{i=1}^{m} 2 T_{*}\left(\varphi_{1}, \ldots, \varphi_{i-1}, f_{i}-\varphi_{i}, f_{i+1}, \ldots, f_{m}\right)>\delta\right\}\right) \\
& \leq \sum_{i=1}^{m} \nu\left(\left\{y \in Y: 2 T_{*}\left(\varphi_{1}, \ldots, \varphi_{i-1}, f_{i}-\varphi_{i}, f_{i+1}, \ldots, f_{m}\right)>\frac{\delta}{m}\right\}\right) \\
& \leq \sum_{i=1}^{m}\left[\left(2 B \frac{m}{\delta}\right)\left\|\varphi_{1}\right\|_{L^{p_{1}} \ldots . . .}\left\|\varphi_{i-1}\right\|_{L^{p_{i-1}}}\left\|f_{i}-\varphi_{i}\right\|_{L^{p_{i}}}\left\|f_{i+1}\right\|_{\left.L^{p_{i+1}} \ldots . . . f_{m} \|_{L^{p_{m}}}\right]^{q}}\right. \\
& \leq\left(2^{m} B \frac{m}{\delta}\right)^{q} \eta^{q} \sum_{i=1}^{m}\left(\prod_{j \neq i}\left\|f_{j}\right\|_{L^{p_{j}}}^{q}\right) .
\end{aligned}
$$

Letting $\eta \rightarrow 0$, we deduce (36). We conclude that $T_{t}[\vec{f}]$ is a Cauchy sequence and hence it converges $\nu$-a.e. to some $T[\vec{f}]$ which satisfies the claimed assertions.

We now prove Corollary 6

Proof. It suffices to prove the assertion for almost all $x$ in a ball $N \mathbb{B}^{n}$, as $\mathbb{R}^{n}$ is a countable union of balls. Let us fix a ball $N \mathbb{B}^{n}$. Then we replace the given $f_{i}$ in $L_{l o c}^{p_{i}}$ by $g_{i}=f_{i} \chi_{(N+1) \mathbb{B}^{n}}$ since $S_{\alpha, t}\left(f_{1}, \ldots, f_{m}\right)(x)=S_{\alpha, t}\left(g_{1}, \ldots, g_{m}\right)(x)$ when $x \in N \mathbb{B}^{n}$ and $0<t<1$. As $g_{i}$ have compact support and lie in $L^{p_{i}}$, they also lie in $L^{q_{i}}$, where $q_{i}<p_{i}$ are chosen so that $\frac{1}{q}=\sum_{i=1}^{m} \frac{1}{q_{i}}<\frac{m n-\alpha}{n}$. As $q_{i}<\infty$, the space of smooth functions with compact support is a dense subspace of $L^{q_{i}}$. Now (16) is easily shown to hold for smooth functions with compact support $f_{i}$, when $0 \leq \alpha \leq 1$, thus (35) holds with $T_{t}=S_{\alpha, t}$. Moreover (34) holds by Theorem 3 if $\alpha<1$ or by [8] if $\alpha=1$. By Proposition 8, for $t<1$, we obtain that for almost all $x \in N \mathbb{B}^{n}$ we have

$$
\lim _{t \rightarrow 0} S_{\alpha, t}^{m}\left(f_{1}, \ldots, f_{m}\right)(x)=\lim _{t \rightarrow 0} S_{\alpha, t}^{m}\left(g_{1}, \ldots, g_{m}\right)(x)=\prod_{j=1}^{m} g_{j}(x)=\prod_{j=1}^{m} f_{j}(x),
$$

thus (16) holds for all $g_{i}$ in $L^{q_{i}}$, in particular for our given $f_{i}$ in $L_{l o c}^{p_{i}}$. 


\section{References}

1. Anderson, T. and Palsson, E., Bounds for discrete multilinear spherical maximal functions in higher dimensions, Bull. Lond. Math. Soc. 53 (2021), 855-860.

2. Anderson, T. and Palsson, E., Bounds for discrete multilinear spherical maximal functions, Preprint, 2019. arXiv:1910.11409 [math.CA]

3. Barrionuevo, J., Grafakos, L., He, D., Honzík, P. and Oliveira, L., Bilinear spherical maximal function, Math. Res. Lett. 25 (2018), 1369-1388.

4. Bourgain, J., Averages in the plane over convex curves and maximal operators, $J$. Anal. Math. 47 (1986), 69-85.

5. Calderon, C. P., Lacunary spherical means, Ill. J. Math. 23 (1979), 476-484.

6. CArbery, A., Radial Fourier multipliers and associated maximal functions, NorthHoll. Math. Stud. 111 (1985), 49-56.

7. Cowling, M. and Mauceri, G., On maximal functions, Milan J. Math. 49 (1979), 79-87.

8. Dosidis, G., Multilinear spherical maximal function, Proc. Amer. Math. Soc. 149 (2021), 1471-1480.

9. Duonndikoetxea, J. and Vega, L., Spherical means and weighted inequalities, $J$. Lond. Math. Soc. 53 (1996), 343-353.

10. Geba, D., Greenleaf, A., Iosevich, A., Palsson, E. and Sawyer, E., Restricted convolution inequalities, multilinear operators and applications, Math. Res. Lett. 20 (2013), 675-694.

11. Grafakos, L., Classical Fourier Analysis, 3rd ed., Graduate Texts in Mathematics 249, Springer, New York, 2014.

12. Grafakos, L., He, D. and Honzík, P., Maximal operators associated with bilinear multipliers of limited decay, J. Anal. Math. 143 (2021), 231-251.

13. Grafakos, L., Liu, L., Lu, S. and Zhao, F., The multilinear Marcinkiewicz interpolation theorem revisited: The behavior of the constant, J. Funct. Anal. 262 (2012), 2289-2313.

14. Greenleaf, A., Principal curvature and harmonic-analysis, Indiana Univ. Math. J. 30 (1981), 519-537.

15. Heo, Y., Hong, S. and Yang, C. W., Improved bounds for the bilinear spherical maximal operators, Preprint, 2019.

16. Iosevich, A. and SAwyer, E., Three problems motivated by the average decay of the Fourier transform, Contemp. Math. 320 (2003), 205-216.

17. Jeong, E. and LEe, S., Maximal estimates for the bilinear spherical averages and the bilinear Bochner-Riesz, J. Funct. Anal. 279 (2020), 108629, 29 pp.

18. Lerner, A., Ombrosi, S., Pérez, C., Torres, R. and Trujillo-González, R., New maximal functions and multiple weights for the multilinear CalderónZygmund theory, Adv. Math. 220 (2009), 1222-1264.

19. Magyar, A., Stein, E. and Wainger, S., Discrete analogues in harmonic analysis: spherical averages, Ann. Math. 155 (2002), 189-208.

20. Mockenhaupt, G., Seeger, A. and Sogge, C. D., Wave front sets, local smoothing and Bourgain's circular maximal theorem, Ann. Math. 136 (1992), 207-218.

21. Oberlin, D., Multilinear convolutions defined by measures on spheres, Trans. Am. Math. Soc. 310 (1988), 821-835. 
22. Rubio de Francia, J.-L., Maximal functions and Fourier transforms, Duke Math. J. 53 (1986), 395-404.

23. Schlag, W., A geometric proof of the circular maximal theorem, Duke Math. J. 93 (1998), 505-534.

24. Stein, E. M., Maximal functions: spherical means, Proc. Nat. Acad. Sci. 73 (1976), $2174-2175$.

25. Stein, E. M., Harmonic Analysis: Real-Variable Methods, Orthogonality, and Oscillatory Integrals, Princeton Mathematical Series 43, Springer, New Jersey, 1993.

26. Stein, E. M. and Strömberg, J. O., Behavior of maximal functions in $R^{n}$ for large n, Ark. Mat. 21 (1983), 259-269.

Georgios Dosidis

Department of Mathematics

Charles University

11636 Praha 1

Prague

Czech Republic

dosidis@karlin.mff.cuni.cz

Received May 5, 2020

in revised form November 16, 2020
Loukas Grafakos

Department of Mathematics

University of Missouri

Columbia MO 65203

U.S.A.

grafakosl@missouri.edu 